\title{
Fault-Tolerant Control of Quadrotor UAVs Based on Back-Stepping Integral Sliding Mode Approach and Iterative Learning Algorithm
}

\author{
Davood Allahverdy ${ }^{(D},{ }^{1}$ Ahmad Fakharian $\mathbb{D}^{1},{ }^{2}$ and Mohammad Bagher Menhaj $\mathbb{D}^{3}$ \\ ${ }^{1}$ Science and Research Branch, Islamic Azad University, Tehran, Iran \\ ${ }^{2}$ Faculty of Electrical, Biomedical, and Mechatronics Engineering, Qazvin Branch, Islamic Azad University, Qazvin, Iran \\ ${ }^{3}$ Department of Electrical Engineering, Amirkabir University of Technology, Tehran, Iran
}

Correspondence should be addressed to Ahmad Fakharian; ahmad.fakharian@qiau.ac.ir

Received 19 March 2021; Revised 15 July 2021; Accepted 18 August 2021; Published 26 August 2021

Academic Editor: Hao Shen

Copyright (c) 2021 Davood Allahverdy et al. This is an open access article distributed under the Creative Commons Attribution License, which permits unrestricted use, distribution, and reproduction in any medium, provided the original work is properly cited.

\begin{abstract}
In this paper, a fault-tolerant control system based on back-stepping integral sliding mode controller (BISMC) is designed and analyzed for both nonlinear translational and rotational subsystems of the quadrotor unmanned aerial vehicles (UAVs). The novelty of this paper is about combination of a classic controller with a repetitive algorithm to reduce the response time to actuator faults and have better tracking performance. The actuator fault is defined based on the loss of effectiveness and bias fault. Next, the iterative learning control algorithm (ILCA) is used to compensate for the unknown fault input according to previous recorded experiences. In the normal condition (without actuators fault), BISMC can force the actual trajectories toward the desired commands and reduce chattering about control signals, and in the presence of the actuators fault or external disturbances, the mentioned learning algorithm can incline the accuracy of the tracking performance and compensate for the occurred error. The Lyapunov theory illustrates that the proposed control strategy can stabilize the system despite the actuators' fault and external disturbances. The simulation results show the effectiveness of the proposed scheme in comparison with another method.
\end{abstract}

\section{Introduction}

Over the last decade, quadrotor UAVs have been noticed for their properties such as speed, small size, and harmless for the environment in weather monitoring, payload transportation, power line inspection, and commercial industries. The dynamic of the quadrotor is highly coupled, nonlinear, and under-actuated, and it is affected by aerodynamic effects and inertial torque during the flight pass. During a flight, one actuator maybe break down or weather conditions may influence the dynamic as an external disturbance so designing a fault-tolerant control system (FTCS) is necessary for quadrotor UAVs.

In recent years, repeatedly, FTCS has been designed based on back-stepping and sliding mode control (SMC). A combination of back-stepping and SMC was used to design a
FTCS where actuator fault was considered as a single-rotor failure [1]. By attending to unmeasured states, actuator fault, control input constraints, and external disturbances, an adaptive back-stepping was designed for quadrotor [2] and a robust FTCS based on constraint back-stepping technique was designed [3]. A robust back-stepping controller with adaptive interval type- 2 fuzzy logic was proposed to control the attitude and position of the modified quadrotor UAVs under actuator faults and external disturbances $[4,5]$. In [6], to cope with wind effect and actuator faults, the decentralized fractional-order (FO) back-stepping adaptive neural fault-tolerant control (FTC) method was presented for the altitude subsystem of UAVs. To ensure fast and global finitetime stability of the UAVs states in the presence of motor failure, a FTCS based on back-stepping terminal sliding mode controller was designed [7]. In [8, 9], a FTCS based on 
a dual adaptive back-stepping method was considered despite the actuator's effectiveness. A new FTCS design according to an adaptive neural network and SMC was presented for quadrotor UAVs where external disturbances and actuator faults were approximated by the neural network [10].

Sliding mode control is a nonlinear control strategy to cope with the uncertainties and external disturbances on the sliding surface [11-14], and this method repeatedly was applied for designing a FTCS for the quadrotor UAVs. In [15], a SMC for quadrotor UAVs was designed to obtain sustained performance in the presence of actuator faults. Based on a nonsingular terminal sliding mode control (NTSMC), finite-time position, and altitude condition, a flight controller was proposed for a quadrotor with a total rotor failure [16]. A fault-tolerant normal adaptive sliding mode control (NASMC) approach was presented to account with uncertainties and actuator faults [17]. A dual adaptive fault-tolerant control strategy for a quadrotor helicopter based on adaptive sliding mode control and adaptive boundary layer was designed despite the unknown actuator's faults and uncertainties [18]. In the presence of the rear servo's stuck fault of tri-rotor [19], a nonlinear FTCS according to an adaptive sliding mode control was presented. A comparative study of SMC, ISMC, and ITSMC based on fault-tolerant controllers for trajectory tracking of the quadrotor UAVs subject to actuator faults was presented $[20,21]$. In [22, 23], an observer based on sliding mode observer (SMO) was utilized to estimate the input saturation and actuator faults, and also a finite-time fault-tolerant controller was presented for quadrotor UAVs. In [24], a fault-tolerant attitude stabilization control problem of a quadrotor UAV was addressed by a FTCS based on an integral-type sliding mode control strategy. By utilizing the fuzzy neural networks (FNNs), the unknown nonlinear fault actuator was estimated, and an adaptive fractional-order fault-tolerant cooperative control based on distributed sliding mode (DSM) was proposed [25]. For multi UAVs despite the rotor failure and input saturation, a distributed finite-time sliding mode observer was used to estimate unknown nonlinear fault terms [26]. In [27], an observer based on SMC was designed and analyzed for a discrete T-S fuzzy model in the presence of communication burden and a logarithmic quantizer that reachability of the prescribed sliding surface was improved. In order to improve the reachability of the sliding surface, a SMC in the discrete form was presented with integral sliding surface [28].

In an attempt to design ILCA for quadrotor UAVs, in [29], to solve the effect of external disturbances and system model uncertainties, generated control input by ILCA was added to PID controller. A developed fault-tolerant iterative learning control law for a class of linear time-delay system with actuator faults was presented in [30]. A cooperative adaptive iterative learning fault-tolerant control algorithm with the radial basis function neural network is proposed for multiple subway trains subject to the time-iteration-dependent actuator faults [31]. In [32], to compensate the systematic modeling errors, the iterative learning algorithm by contribution of feed forward controller was used until the error of states was zero. To reject the external disturbances and improve the accuracy of performance, ILC was used for control of multiquadrotor where each vehicle learns from the experience of its own and its neighbours' previous task repetitions [33].

According to the previous references, some challenges occurred during actuator faults such as constant tracking errors, long time to force states toward references trajectory, and the effect of external disturbances on the performance of the quadrotor. The contribution of this work is to design a FTCS for the quadrotor UAVs despite the actuator faults by combination of a classic controller and an online repetitive algorithm; therefore, the mentioned control strategy can reduce the response time to actuator faults and have better tracking performance. The loss of effectiveness and bias faults are considered as actuator faults. ILCA is used to compensate the unknown fault input based on online and simple formulas in each iteration where the next unknown fault inputs are generated according to previous results [34] as a control input. A FTCS is designed and analyzed based on BISMC for quadrotor UAVs. Finally, in a flight motion in the presence of the actuator failures, the generated inputs based on ILCA and BISMC are added to calculate the total control input. The Lyapunov theory is utilized to justify the stability of the system. The results show that the proposed method (ILCA) has better performance in comparison with another method, and the mentioned control strategy has better tracking, accuracy, and robustness.

The remainder of the paper is organized as follows. Section 2 illustrates the quadrotor UAVs dynamics despite the actuator faults and external disturbances. ILCA to compensate the effect of the unknown fault inputs and external disturbances is designed in Section 3. The FTCS based on BISMC and stability study are presented in Section 4. Simulation results are presented in Section 5, and finally Section 6 concludes the paper.

1.1. Notation. Any vector or matrix is presented with bold letter. $R^{n}$ refers to the $n$-dimensional Euclidean space and $R^{m \times n}$ is real matrix by $m \times n$ size. All the mode equations are time dependent. The state vector is introduced by $x$. The first and second time derivations of the states vector are cited, respectively, as $\dot{x}$ and $\ddot{x}$.

\section{Quadrotor UAVs with Actuator Faults}

In this section, at first, the full nonlinear dynamic of the quadrotor UAVs by considering the external disturbances and without actuators fault is given [35] as follows:

$$
\begin{aligned}
& \ddot{x}=(\sin \theta \cos \varphi \cos \psi+\sin \varphi \sin \psi)\left(\frac{b}{m}\right) u_{1}-\frac{d_{x}}{m}, \\
& \ddot{y}=(\cos \varphi \sin \theta \cos \psi+\sin \varphi \sin \psi)\left(\frac{b}{m}\right) u_{1}-\frac{d_{y}}{m},
\end{aligned}
$$




$$
\begin{aligned}
& \ddot{z}=-g+\cos \theta \cos \varphi\left(\frac{b}{m}\right) u_{1}-\frac{d_{z}}{m}, \\
& \ddot{\varphi}=\dot{\theta} \dot{\psi} \frac{I_{y}-I_{z}}{I_{y}}+\dot{\theta} \Omega_{r} \frac{J_{r}}{I_{x}}+\frac{L b}{I_{x}}+u_{2}-\frac{d_{\varphi}}{I_{x}}, \\
& \ddot{\theta}=\dot{\varphi} \dot{\psi} \frac{I_{z}-I_{x}}{I_{y}}+\dot{\varphi} \Omega_{r} \frac{\mathrm{J}_{r}}{I_{y}}+\frac{L b}{I_{y}}+u_{3}-\frac{d_{\theta}}{I_{y}}, \\
& \ddot{\Psi}=\dot{\varphi} \dot{\theta} \frac{I_{x}-I_{y}}{I_{z}}+\frac{d}{I_{z}}+u_{4}-\frac{d_{\Psi}}{I_{z}},
\end{aligned}
$$

where $x, y$, and $z$ are relating to the translational subsystem and $\varphi, \theta$, and $\psi$ denote the roll, pitch, and yaw angle of the rotational subsystem of the quadrotor UAVs.

$d_{x}, d_{y}, d_{z}, d_{\varphi}, d_{\theta}$, and $d_{\psi}$ are external disturbances. $I_{x}, I_{y}$, and $I_{z}$ are entitled as moments of inertia of the quadrotor to the corresponding axes. $g$ is the gravitational constant, $m$ is the total mass of the quadrotor, $L$ is the distance from the center of the quadrotor to the center of propeller, $J_{r}$ is the inertia relating to the rotor, and $b$ and $d$ are the thrust and drag coefficients, respectively. $u_{1}, u_{2}, u_{3}$, and $u_{4}$ are the control inputs that produce thrust, rolling torque, pitching torque, and yawing torque, respectively, in the rotor that are described as follows:

$$
\begin{aligned}
& u_{1}=\Omega_{1}^{2}+\Omega_{2}^{2}+\Omega_{3}^{2}+\Omega_{4}^{2}, \\
& u_{2}=-\Omega_{2}^{2}+\Omega_{4}^{2}, \\
& u_{3}=-\Omega_{3}^{2}, \\
& u_{4}=\Omega_{1}^{2}-\Omega_{2}^{2}+\Omega_{3}^{2}-\Omega_{4}^{2} .
\end{aligned}
$$

The definition of quadrotor dynamic parameters in equations (1)-(6) is cited in Table 1.

The quadrotor UAVs structure is shown in Figure 1.

The structure of the quadrotor consists of four rotors that each of them can generate thrust and torque for each direction movement. So, in a flight motion, actuators fault or rotors failure may occur or bad weather conditions may influence the dynamic as external disturbances. In this study, the loss of effectiveness and bias fault for the control signals of the main dynamic equations (1)-(6) are modeled as follows:

$$
u_{i}=E \delta_{i}+\zeta_{i}, \quad \text { for } i=1,2,3,4,
$$

where $\delta_{i}=\left[\delta_{1}, \delta_{2}, \delta_{3}, \delta_{4}\right]^{T}$ is relating to command control vector, $\zeta_{i}=\left[\zeta_{1}, \zeta_{2}, \zeta_{3}, \zeta_{4}\right]^{T}$ is bias faults, and $E_{i}=\operatorname{diag}\left\{E_{1}, E_{2}, E_{3}, E_{4}\right\} \in(\bar{E}, 1]$ is the rotor effectiveness failure matrix with $\bar{E}$ as a known lower bound to keep the
TABle 1: Parameters of the dynamic.

\begin{tabular}{lcc}
\hline Parameters & Value & Unit \\
\hline$L$ (arm length) & 0.232 & $\mathrm{M}$ \\
$b$ (thrust coefficient) & $3.13 \times 10^{-5}$ & $\mathrm{n} \cdot \mathrm{s}^{2}$ \\
$d$ (drag coefficient) & $7.5 \times 10^{-7}$ & $\mathrm{~m} \cdot \mathrm{s}^{2}$ \\
$m$ (mass of quadrotor) & 0.52 & $\mathrm{~kg}$ \\
$I_{x}$ (moment of inertia) & $6.228 \times 10^{-3}$ & $\mathrm{~kg} \cdot \mathrm{m}^{2}$ \\
$I_{y}$ (moment of inertia) & $6.228 \times 10^{-3}$ & $\mathrm{~kg} \cdot \mathrm{m}^{2}$ \\
$I_{z}$ (moment of inertia) & $1.121 \times 10^{-2}$ & $\mathrm{~kg} \cdot \mathrm{m}^{2}$ \\
$J_{r}$ (rotor inertia) & $6 \times 10^{-5}$ & $\mathrm{~kg} \cdot \mathrm{m}^{2}$ \\
$\Omega_{r}$ (speed component) & 297 & $\mathrm{rad} / \mathrm{s}$ \\
\hline
\end{tabular}

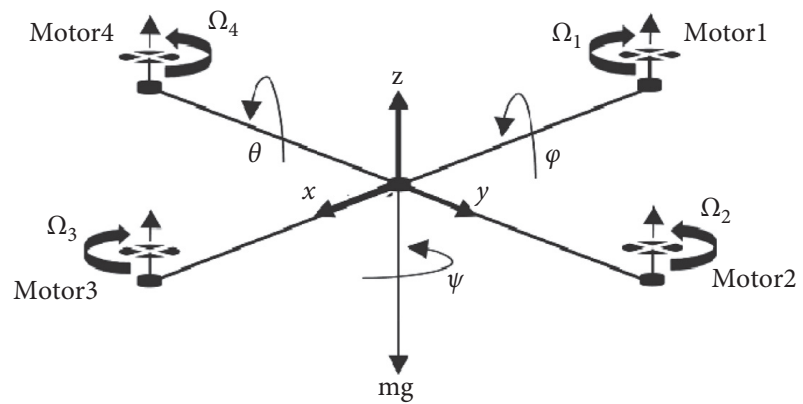

FIgURE 1: Quadrotor UAVs structure.

controllability of the quadrotor UAVs. It should be taken into consideration that when $E_{i}=1$ and $\zeta_{i}=0$, it means that the rotors work without any problem and failure, and $0<\bar{E}<E_{i}<1, \quad \zeta_{i} \neq 0$ illustrates that the $i_{\text {th }}$ the rotor is working with low effectiveness and bias fault.

Remark 1. Constants of the rotor effectiveness and bias failures are known, but the lower bound as $\bar{E}$ should be defined to maintain controllability and avoid actuator saturation of the quadrotor after the occurrence of the fault. This limitation can be calculated based on rotor and quadrotor physical parameters so that the following inequality condition is satisfied:

$$
\bar{E}>\left(\frac{1}{2 \Omega_{\max }}\right)\left(\frac{m g}{b}\right),
$$

where $\Omega_{\max }$ is the maximum rotor velocity limit. It should be taken into consideration that $\bar{E}$ can be reduced by changing the total quadrotor mass or employing another rotor with a higher velocity saturation limit.

By considering actuator fault as equation (8), the dynamic of quadrotor UAVs equations (1)-(6) by considering states vector as $X_{i}=[x, y, z, \varphi, \theta, \psi, \dot{x}, \dot{y}, \dot{z}, \dot{\varphi}, \dot{\theta}, \dot{\Psi}]$ can be rewritten in the following form: 


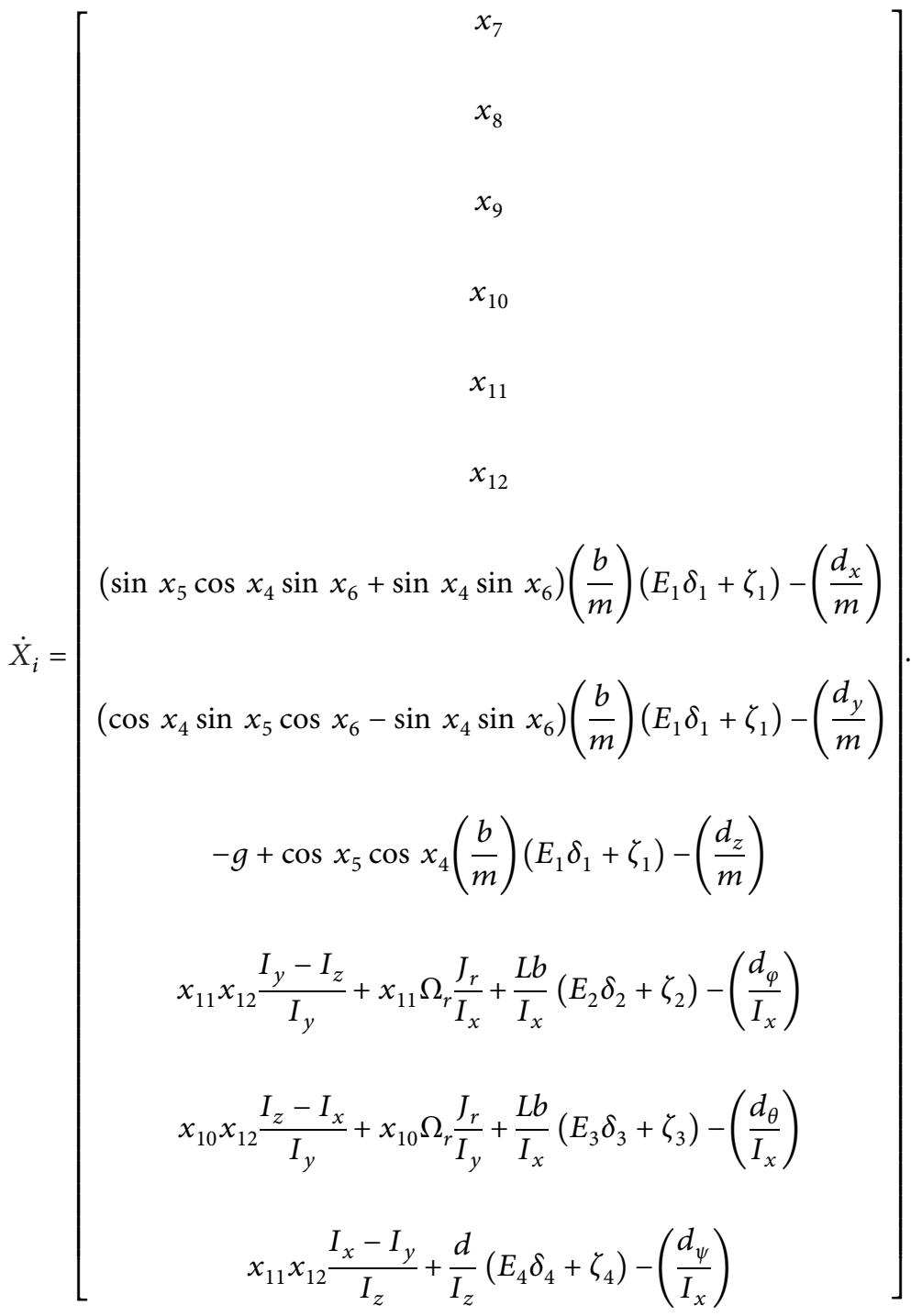

\section{Actuator Faults Estimation Based on ILCA}

To incline the accuracy of tracking performance and reject the external disturbances during a flight motion, machine learning control is designed and analyzed repeatedly for quadrotor UAV [36]. ILCA is a powerful method for approximating the uncertainties of nonlinear dynamics [37]. The mentioned learning algorithm is proposed to solve the repeated tasks in each iteration, and it runs until the errors of states are zero [38]. ILCA is a more accurate method in comparison with other approximation methods because it is an online method that the next unknown control input is calculated based on recorded experiments in the previous iterations. The main contribution of ILCA is to calculate the control signal as unknown fault input $U_{d}$. Next, $U_{d i}$ is combined with $u_{i}$ for calculating the total control input. In this paper, ILCA is used to estimate the loss of effectiveness fault and bias fault for the quadrotor dynamic. The control system schematic diagram is shown in Figure 2.

The states for designing ILCA is selected as the following vector:

$$
\left[\begin{array}{c}
\dot{Z} \\
\dot{\varphi} \\
\dot{\theta} \\
\dot{\psi}
\end{array}\right]=\left[\begin{array}{c}
-g+\cos \theta \cos \varphi\left(\frac{1}{m}\right) u_{1}-\frac{d_{z}}{m} \\
\dot{\theta} \dot{\frac{I_{y}}{m}} \frac{I_{z}}{I_{y}}+\dot{\theta} \Omega_{r} \frac{J_{r}}{I_{x}}+\frac{1}{I_{x}} u_{2}-\frac{d_{\varphi}}{I_{x}} \\
\dot{\varphi} \dot{\psi} \frac{I_{z}-I_{x}}{I_{y}}+\dot{\varphi} \Omega_{r} \frac{J_{r}}{I_{y}}+\frac{1}{I_{x}} u_{3}-\frac{d_{\theta}}{I_{x}} \\
\dot{\theta} \dot{\psi} \frac{I_{x}-I_{y}}{I_{z}}+\frac{1}{I_{z}} u_{4}-\frac{d_{\psi}}{I_{x}}
\end{array}\right] .
$$

It should be taken into consideration that without actuator fault, BISMC can track the reference commands as follows:

$$
X_{\text {ref }}=f\left(U_{\text {BISMC }}\right) .
$$

The formulation of ILCA for the next iteration is described as follows: 


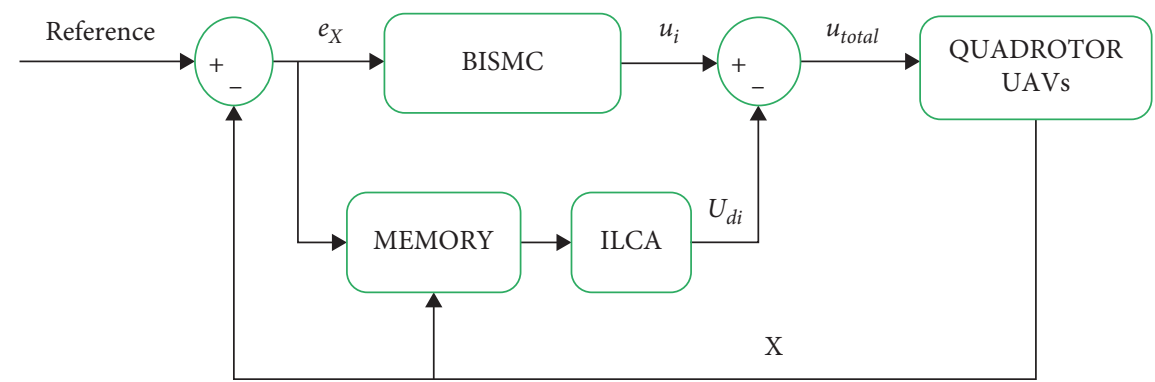

Figure 2: Control system schematic diagram.

$$
U_{d i, t+1}=U_{d i, t}-F^{T} K_{i} e_{X}, \quad \text { for } i=1,2,3,4,
$$

where $U_{d i, 0}$ is the initial input, $K_{i}$ is the positive learning gains, and $e_{X}$ is considered as an error vector as follows:

$$
e_{X i}=X_{i}-X_{\text {ref }, i},
$$

where $F$ is a learning rate matrix along the desired trajectories. According to equation (11) and the linear timevarying method, $F$ is written as follows:

$$
F=\left[\begin{array}{ccc}
\frac{\mathrm{d} X_{9}}{\mathrm{~d} u_{1}} & \cdots & \frac{\mathrm{d} X_{9}}{\mathrm{~d} u_{4}} \\
\vdots & \ddots & \vdots \\
\frac{\mathrm{d} X_{12}}{\mathrm{~d} u_{1}} & \cdots & \frac{\mathrm{d} X_{12}}{\mathrm{~d} u_{4}}
\end{array}\right]
$$

In the presence of the actuator faults, the total control input is written based on unknown fault input $U_{d i}$ and $u_{i}$ as follows:

$$
U_{\text {total }, i}=u_{i}+U_{d i} .
$$

There are two cases in the FTCS of this paper as follows:

(a) If the quadrotor flight is in the normal condition, it means that there are no faults and external disturbances, and the total control input is calculated based on the BISMC as follows:

$$
\begin{aligned}
U_{\text {total }, i} & =u_{i}, \\
\dot{X}_{i} & =f\left(X_{i}\right)+P_{i} g\left(X_{i}\right) U_{\text {total }}=u_{i}, \\
\dot{X}_{i} & =f\left(X_{i}\right)+P_{i} g\left(X_{i}\right)\left(u_{i}\right) .
\end{aligned}
$$

(b) If the quadrotor is not in the normal condition, it means that there are loss of effectiveness and bias fault and external disturbances, and the total control input is calculated based on the BISMC and unknown fault input as follows:

$$
\begin{aligned}
U_{\text {total }} & =u_{i}+U_{d i}, \\
\dot{X}_{i} & =f\left(X_{i}\right)+P_{i} g\left(X_{i}\right) U_{\text {total }}, \\
\dot{X}_{i} & =f\left(X_{i}\right)+P_{i} g\left(X_{i}\right)\left(u_{i}+U_{d i}\right),
\end{aligned}
$$

where $P_{i}$ is the coefficient matrix. Then, equation (17) is rewritten in the following form around the ideal operating point of $u_{i}$ :

$$
\dot{X}_{i} \sim f\left(X_{i}\right)+P_{i} g\left(X_{i}\right)\left(u_{i}\right)+P_{i}\left(\left.\frac{\mathrm{d}}{\mathrm{d} U} g\right|_{u_{i}}\right) \cdot U_{d i} \cdot
$$

The error vector equation (14) can be rewritten as a function of the unknown failure input as follows:

$$
e_{X i}=X_{i}-X_{\mathrm{ref}, i}=P_{i}\left(\left.\frac{\mathrm{d}}{\mathrm{d} U} g\right|_{u_{i}}\right) \cdot U_{d i}=P_{i} \cdot F U_{d i} .
$$

To analyze the stability of the mentioned ILCA, that is, the convergence of the learned input as the number of iterations goes to infinity, lifted-system in vector format is deployed [39]. The system dynamic and the updating rule can be presented as follows:

$$
\begin{aligned}
& {\left[\begin{array}{c}
\dot{X}_{3} \\
\dot{X}_{4} \\
\dot{X}_{5} \\
\dot{X}_{6}
\end{array}\right]=f\left(X_{i}\right)+g\left(X_{i}\right) P_{i}\left[\left[\begin{array}{l}
u_{1} \\
u_{2} \\
u_{3} \\
u_{4}
\end{array}\right]+\left[\begin{array}{l}
U_{d 1} \\
U_{d 2} \\
U_{d 3} \\
U_{d 4}
\end{array}\right],\right.} \\
& {\left[\begin{array}{l}
U_{d 1, t+1} \\
U_{d 2, t+1} \\
U_{d 3, t+1} \\
U_{d 4, t+1}
\end{array}\right]=\left[\begin{array}{l}
U_{d 1, t} \\
U_{d 2, t} \\
U_{d 3, t} \\
U_{d 4, t}
\end{array}\right]-F^{T} K_{i}\left[\begin{array}{l}
e_{X 3} \\
e_{X 4} \\
e_{X 5} \\
e_{X 6}
\end{array}\right] .}
\end{aligned}
$$

By considering $P_{i}$ and $K_{i}$ as lower-triangular, Toeplitz matrices, we have

$$
\begin{gathered}
P_{i}=\left[\begin{array}{llll}
P_{1} & 0 & 0 & 0 \\
P_{2} & P_{1} & 0 & 0 \\
P_{3} & P_{2} & P_{1} & 0 \\
P_{4} & P_{3} & P_{2} & P_{1}
\end{array}\right], \\
K_{i}=\left[\begin{array}{cccc}
K_{1} & 0 & 0 & 0 \\
K_{2} & K_{1} & 0 & 0 \\
K_{3} & K_{2} & K_{1} & 0 \\
K_{4} & K_{3} & K_{2} & K_{1}
\end{array}\right] .
\end{gathered}
$$

By the combination of equations (23) and (24) with equations (21) and (22), we have 


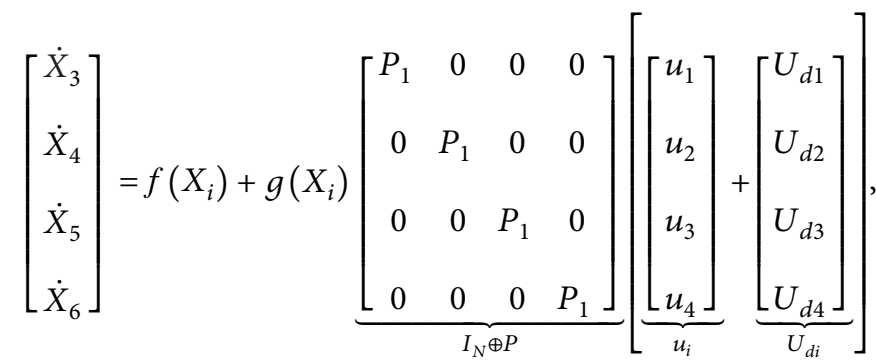

$$
\begin{aligned}
& \underbrace{\left[\begin{array}{c}
U_{d 1, t+1} \\
U_{d 2, t+1} \\
U_{d 3, t+1} \\
U_{d 4, t+1}
\end{array}\right]}_{U_{d i, t+1}}=\underbrace{\left[\begin{array}{c}
U_{d 1, t} \\
U_{d 2, t} \\
U_{d 3, t} \\
U_{d 4, t}
\end{array}\right]}_{U_{d i, t}}-\underbrace{\left[\begin{array}{ccc}
\frac{\mathrm{d} X_{9}}{\mathrm{~d} u_{1}} & \cdots & \frac{\mathrm{d} X_{9}}{\mathrm{~d} u_{4}} \\
\vdots & \ddots & \vdots \\
\frac{\mathrm{d} X_{12}}{\mathrm{~d} u_{1}} & \cdots & \frac{\mathrm{d} X_{12}}{\mathrm{~d} u_{4}}
\end{array}\right]}_{F} \underbrace{\left[\begin{array}{cccc}
K_{1} & 0 & 0 & 0 \\
0 & K_{1} & 0 & 0 \\
0 & 0 & K_{1} & 0 \\
0 & 0 & 0 & K_{1}
\end{array}\right]}_{I_{N} \oplus K_{i}} \underbrace{\left[\begin{array}{c}
e_{X 3} \\
e_{X 4} \\
e_{X 5} \\
e_{X 6}
\end{array}\right],}_{e_{x i}},
\end{aligned}
$$

where $\oplus$ denotes the Kronecker product and $I_{N}$ with $(N \times$ $N)$ is the identity matrix. Using the error definition equation (14) with equation (26),

$$
\underbrace{\left[\begin{array}{c}
U_{d 1, t+1} \\
U_{d 2, t+1} \\
U_{d 3, t+1} \\
U_{d 4, t+1}
\end{array}\right]}_{U_{d i, t+1}}=\underbrace{\left[\begin{array}{c}
U_{d 1, t} \\
U_{d 2, t} \\
U_{d 3, t} \\
U_{d 4, t}
\end{array}\right]}_{U_{d i, t}} \underbrace{\left[\begin{array}{ccc}
\frac{\mathrm{d} X_{9}}{\mathrm{~d} u_{1}} & \cdots & \frac{\mathrm{d} X_{9}}{\mathrm{~d} u_{4}} \\
\vdots & \ddots & \vdots \\
\frac{\mathrm{d} X_{12}}{\mathrm{~d} u_{1}} & \ldots & \frac{\mathrm{d} X_{12}}{\mathrm{~d} u_{4}}
\end{array}\right]}_{F} \underbrace{\left[\begin{array}{cccc}
K_{1} & 0 & 0 & 0 \\
0 & K_{1} & 0 & 0 \\
0 & 0 & K_{1} & 0 \\
0 & 0 & 0 & K_{1}
\end{array}\right]}_{I_{N}^{\oplus K_{i}}} \underbrace{\left[\begin{array}{c}
X_{3} \\
X_{4} \\
X_{5} \\
X_{6}
\end{array}\right]}_{X_{i}} \underbrace{\left[\begin{array}{c}
X_{\mathrm{ref}, 3} \\
X_{\mathrm{ref}, 4} \\
X_{\mathrm{ref}, 5} \\
X_{\mathrm{ref}, 6}
\end{array}\right]}_{X_{\mathrm{ref}, i}} .
$$

Equation (28) can be rewritten as following form:

$$
U_{d i, t+1}=U_{d i, t}-F^{T}\left(I_{N} \oplus K_{i}\right)\left(X_{i}-X_{\mathrm{ref}, i}\right) .
$$

Replacing equation (20) into equation (28),

$$
U_{d i, t+1}=U_{d i, t}-F^{T} F P_{i}\left(I_{N} \oplus K_{i}\right)\left(U_{d i}\right) .
$$

Definition 1. The ILC is asymptotically stable if there exist $\bar{U}_{i} \cdot\left|U_{\text {total }, i}\right|<\bar{U}_{i}$ and $\lim _{t \rightarrow \infty} U_{\text {total }, i}$.

\section{Faults Tolerant Control System Design}

In this section, a FTCS based on BISMC is designed for quadrotor UAVs despite the actuator faults. The dynamic of the system with rotor failures is considered as equation (11). The combination of back-stepping and integral sliding mode control can force the state's error to zero and incline the accuracy of the tracking [40]. Next, the Lyapunov theory is employed to justify the stability of the subsystems.

$z_{3}=x_{3}-x_{3 d}$ is defined as altitude error, and the time derivation can be written as $\dot{z}_{3}=\dot{x}_{3}-\dot{x}_{3 d}$. By considering positive Lyapunov function and the time derivation as $v_{1}=$ $(1 / 2) z_{3}^{2}$ and $\dot{v}_{1}=z_{3} \dot{z}_{3}$ respectively, we have

$$
\dot{v}_{1}=z_{3}\left(\dot{x}_{3}-\dot{x}_{3 d}\right)
$$

Define $z_{9}=\dot{x}_{3}-a_{1}$, where $a_{1}$ is a virtual control input. Then, $\dot{v}_{1}$ can be rewritten as follows:

$$
\dot{v}_{1}=z_{3} z_{9}+z_{3}\left(a_{1}-\dot{x}_{3 d}\right) \text {. }
$$

To make the stability of equation (31), $a_{1}$ is defined as follows:

$$
a_{1}=\dot{x}_{3 d}-c_{1} z_{3}
$$

where $c_{1}>0$. Next, by replacing equation (32) into equation (31), $\dot{v}_{1}$ can be computed as follows:

$$
\dot{v}_{1}=z_{3} z_{9}+z_{3}\left(\dot{x}_{3 d}-c_{1} z_{3}-\dot{x}_{3 d}\right)=-c_{1} z_{3}^{2}+z_{3} z_{9} \text {. }
$$

The integral sliding mode surface for the translation subsystem is selected as follows: 


$$
s_{1}=z_{9}+k_{1} \int z_{3}
$$

By the combination of the switching law as $\dot{s}_{1}=-\varepsilon s_{1}-$ $\operatorname{sgn}\left(s_{1}\right)$ with the time derivation of equation (34),

$$
\dot{z}_{9}+k_{1} z_{3}=-\varepsilon s_{1}-\operatorname{sgn}\left(s_{1}\right) .
$$

Replacing equation (33) with equation (35), the control torque $\delta_{1}$ is calculated as follows:

$$
\delta_{1}=\frac{m}{e_{1} \cos x_{5} \cos x_{4} b}\left[-\left(k_{1}+c_{1}\right) z_{3}+g-\varepsilon s_{1}-\operatorname{sgn}\left(s_{1}\right)+\dot{x}_{9 d}+\frac{d_{z}}{m}\right]-\frac{U_{1 d}}{e_{1}}-\frac{\zeta_{1}}{e_{1}} .
$$

Theorem 1. By considering the calculated control command in equation (35), the $z$ position response will be driven to a certain bounded region around sliding surface equation (33).

Proof. A positive Lyapunov function is defined for stability analysis as follows:

$$
v_{2}=\frac{1}{2} v_{1}^{2}+\frac{1}{2} s_{1}^{2} .
$$

The time derivation of equation (37) is calculated as follows:

$$
\dot{v}_{2}=v_{1} \dot{v}_{1}+s_{1} \dot{s}_{1}
$$

and

$$
\dot{v}_{2}=v_{1} \dot{v}_{1}+s_{1}\left(-g+\cos x_{5} \cos x_{4}\left(\frac{b}{m}\right)\left(e_{1} \delta_{1}+\zeta_{1}+U_{d}\right)-\frac{d_{z}}{m}\right)
$$

By replacing equation (36) into equation (39), it can be concluded that the necessary condition of the Lyapunov theorem is verified $\left(\dot{v}_{2}<0\right)$ and the state trajectory can be driven to a certain bounded region around the sliding surface.

Designing a fault-tolerant control system (FTCS) for the rotational subsystem is similar to the translational subsystem. More definition about calculating $\delta_{2}, \delta_{3}$, and $\delta_{4}$ is presented in Appendix. The control torques $\delta_{2}, \delta_{3}$, and $\delta_{4}$ are designed as follows:

$$
\begin{aligned}
& \delta_{2}=\frac{I_{x}}{L b}\left[-\left(k_{2}+c_{2}\right) z_{4}-\varepsilon s_{2}-\operatorname{sgn}\left(s_{2}\right)+\dot{x}_{10 d}+\frac{d_{\varphi}}{I_{x}}-x_{11} \Omega_{r} \frac{J_{r}}{I_{x}}-x_{11} x_{12} \frac{I_{y}-I_{z}}{I_{y}}\right]-\frac{U_{2 d}}{e_{2}}-\frac{\zeta_{2}}{e_{2}}, \\
& \delta_{3}=\frac{I_{y}}{L b}\left[-\left(k_{3}+c_{3}\right) z_{5}-\varepsilon s_{3}-\operatorname{sgn}\left(s_{3}\right)+\dot{x}_{11 d}+\frac{d_{\theta}}{I_{y}}-x_{10} \Omega_{r} \frac{J_{r}}{I_{y}}-x_{10} x_{12} \frac{I_{z}-I_{x}}{I_{y}}\right]-\frac{U_{3 d}}{e_{3}}-\frac{\zeta_{3}}{e_{3}}, \\
& \delta_{4}=\frac{I_{z}}{d}\left[-\left(k_{4}+c_{4}\right) z_{6}-\varepsilon s_{4}-\operatorname{sgn}\left(s_{4}\right)+\dot{x}_{12 d}+\frac{d_{\Psi}}{I_{z}}-x_{11} x_{12} \frac{I_{x}-I_{y}}{I_{z}}\right]-\frac{U_{4 d}}{e_{4}}-\frac{\zeta_{4}}{e_{4}} .
\end{aligned}
$$

\section{Simulation}

In this section, the performance of the proposed control strategy is evaluated in comparison with [10] that used a FTCS based on SMC and neural network. The initial states for the rotational and the translational subsystem are considered as follows: $[x, y, z]=[0.4,0.7,0]$ and $[\varphi, \theta, \psi]=[0,0,-0.12]$. The controller parameters are selected as follows: $k_{i}=100$ for $i=1,2,3,4$ and $K, \varepsilon, c_{1}, c_{2}, c_{3}, c_{4}>0$. The parameters of the quadrotor UAVs dynamic are listed in Table 1. The external disturbances are defined as follows:

$$
\begin{aligned}
& d_{x}=2 \sin t, d_{y}=2 \sin t, d_{z}=2 \sin t, \\
& d_{\varphi}=0.1 \sin \left(\frac{2 \pi}{50} t\right), d_{\theta}=0.1 \sin \left(\frac{2 \pi}{50} t\right), \\
& d_{\Psi}=0.1 \sin \left(\frac{2 \pi}{50} t\right) .
\end{aligned}
$$

The desired trajectories for the rotational subsystem are defined as follows: $x_{1 d}=0.2 * \sin (\pi t), \quad x_{2 d}=0.2 *$ $\cos (\pi t)$, and $x_{3 d}=0.1 * t$, and reference commands for the translational subsystem are selected as follows [31]:

$$
\begin{aligned}
& x_{4 d}=\cos ^{-1}\left(\frac{m k_{x} e_{x}}{b u_{1} \sin x_{5 d} \cos x_{6 d}}-\frac{\sin x_{4 d} \sin x_{6 d}}{\sin x_{5 d} \cos x_{6 d}}\right), \\
& x_{5 d}=\sin ^{-1}\left(\frac{m k_{y} e_{y}}{b u_{1} \cos x_{4 d} \cos x_{6 d}}+\frac{\sin x_{4 d} \sin x_{6 d}}{\cos x_{4 d} \cos x_{6 d}}\right) .
\end{aligned}
$$

The unknown actuator loss of effectiveness fault and bias fault occurred at 30 seconds which is described as follows:

$$
\begin{aligned}
E & = \begin{cases}\operatorname{diag}\{1,1,1,1\}, & 0<t<30, \\
\operatorname{diag}\{0.4,1,1,1\}, & \text { else, }\end{cases} \\
\zeta & = \begin{cases}{[0,0,0,0]^{T},} & 0<t<30, \\
{[0.6,0,0,0]^{T},} & \text { else. }\end{cases}
\end{aligned}
$$

The actuator fault influences the controller $u_{1}$ at $t=30 \mathrm{~s}$ by a bias fault $e_{1}=0.4$ and a loss of effectiveness with 


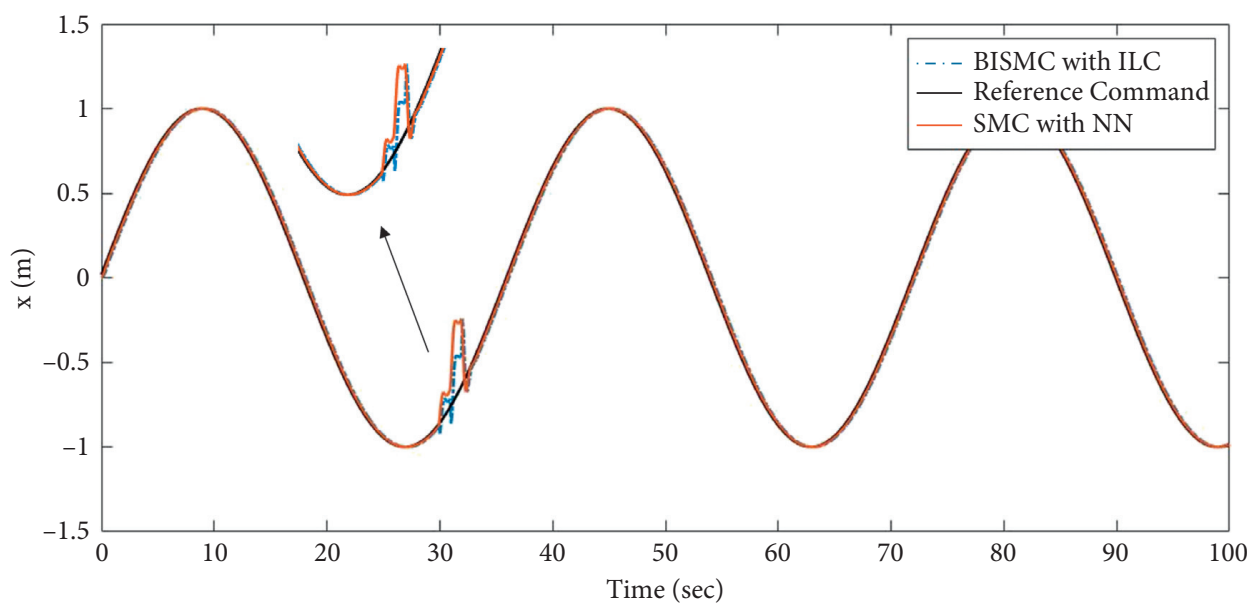

Figure 3: Position $X(\mathrm{M})$.

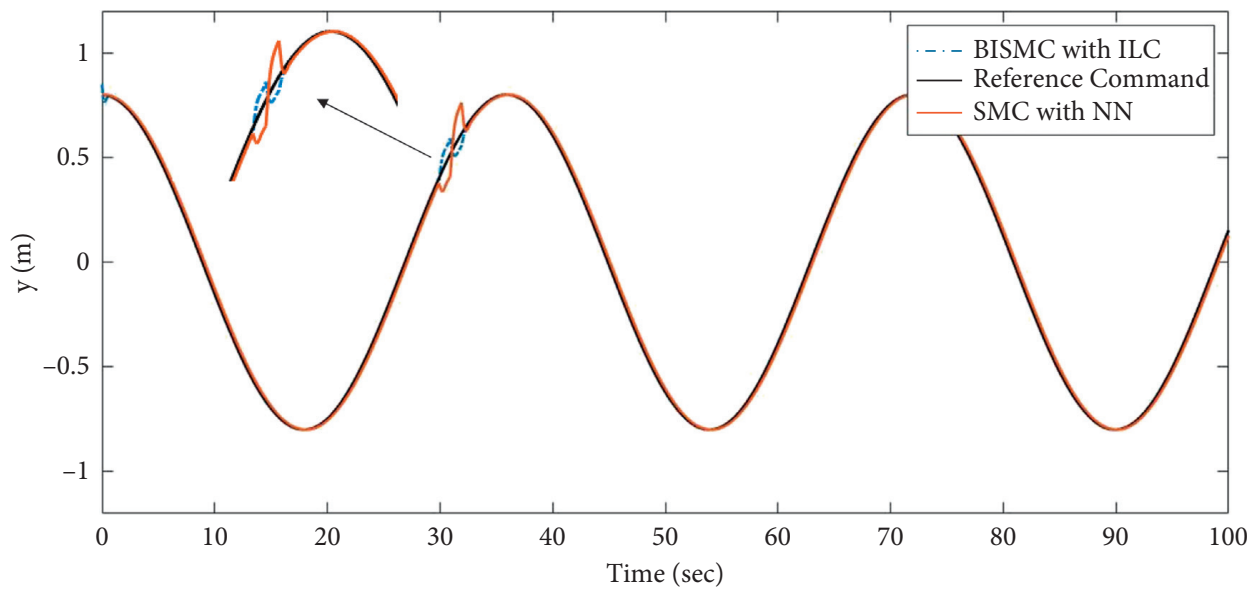

Figure 4: Position $Y(\mathrm{M})$.

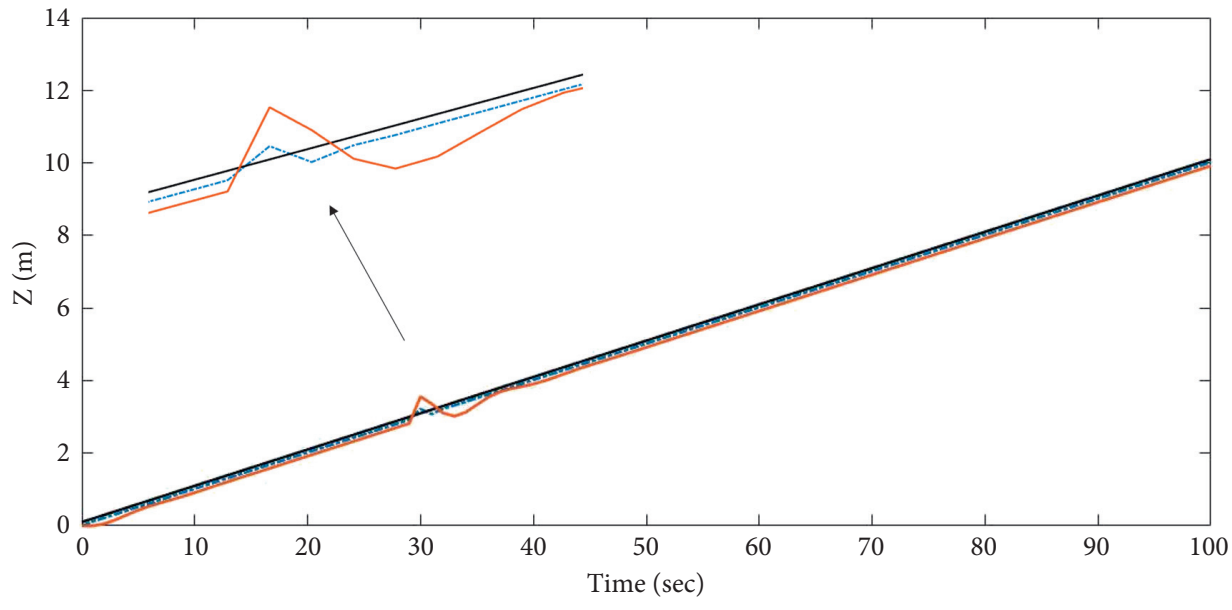

BISMC with ILC

Reference Command

_ SMC with NN

Figure 5: Position $Z$ (M). 


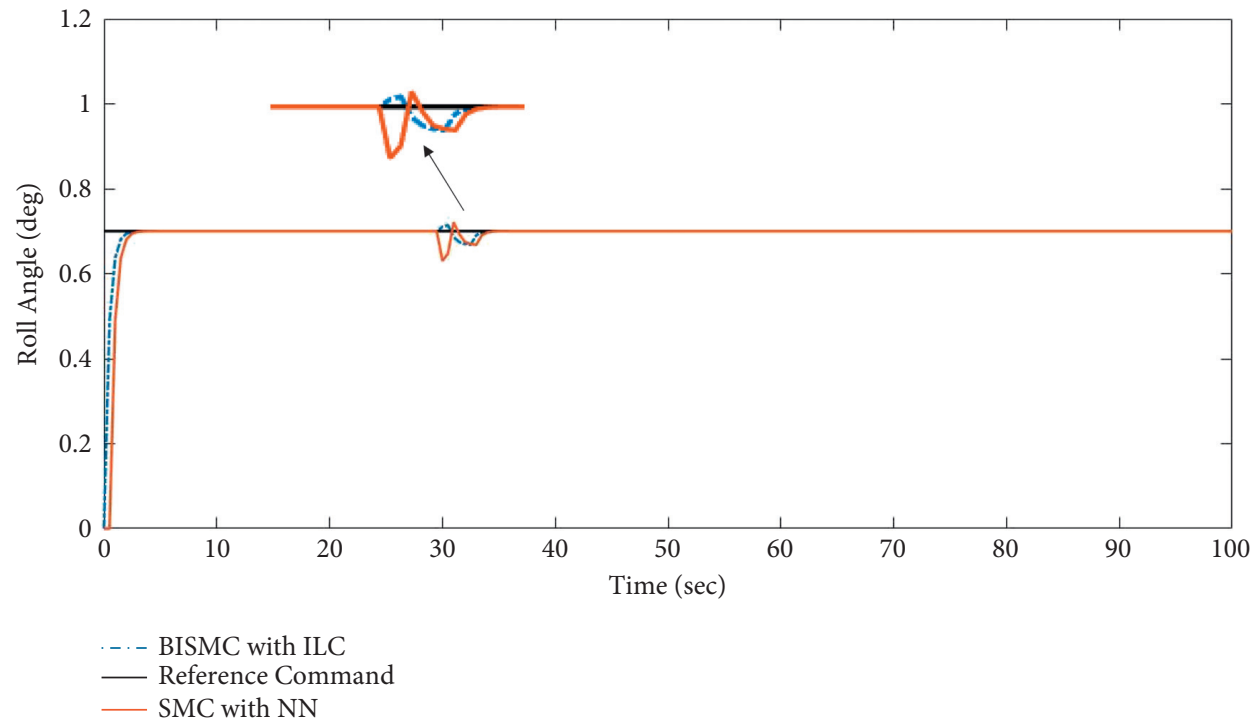

Figure 6: Roll angle (deg).

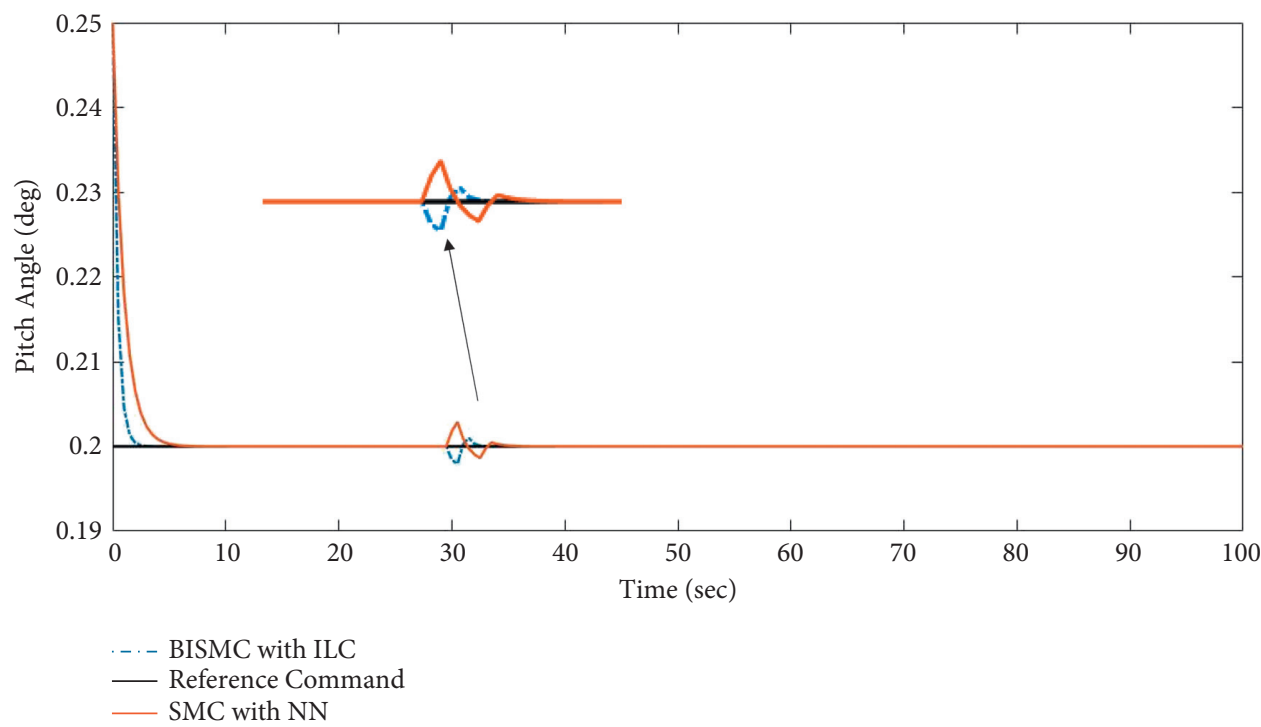

Figure 7: Pitch angle (deg).

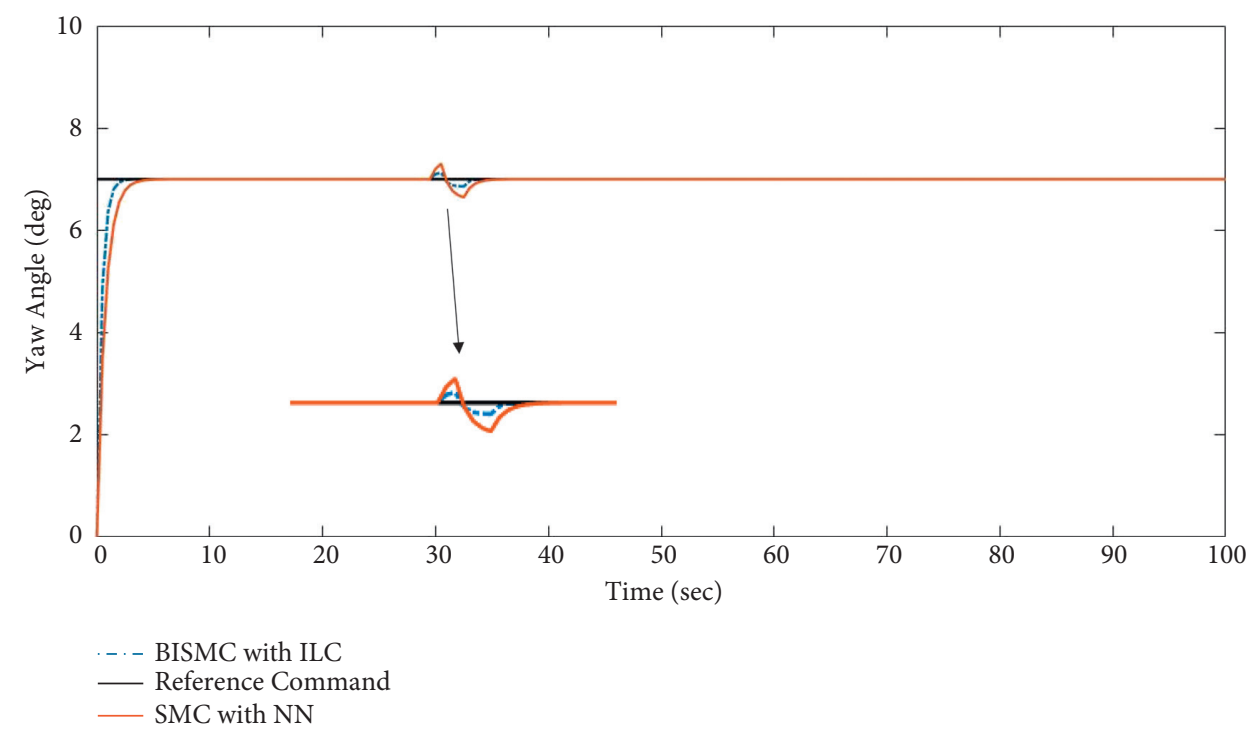

Figure 8: Yaw angle (deg). 


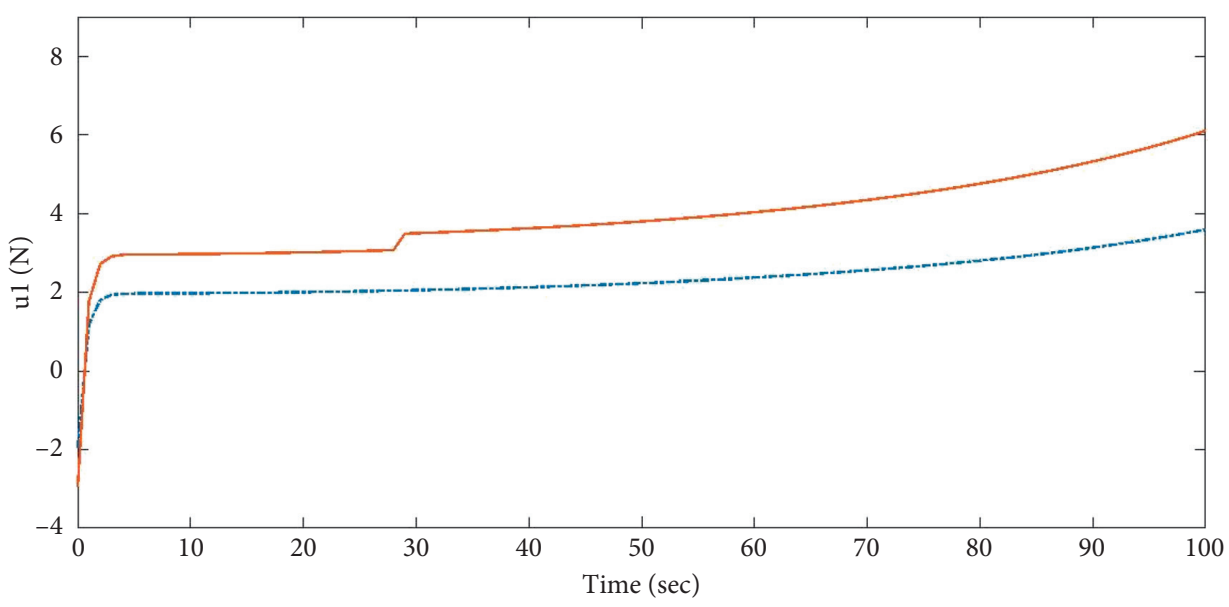

. . - BISMC with ILC

— SMC with NN

FIgURE 9: Control input $u_{1}(\mathrm{~N})$.

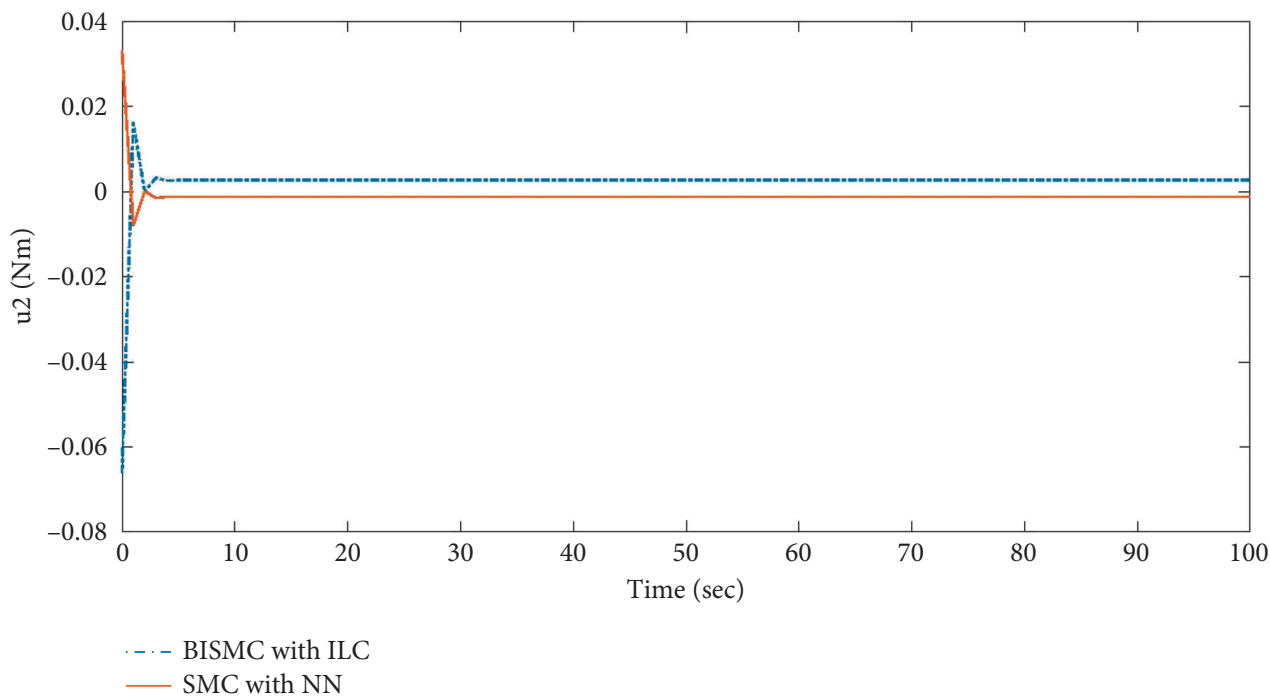

Figure 10: Control input $u_{2}(\mathrm{~nm})$.

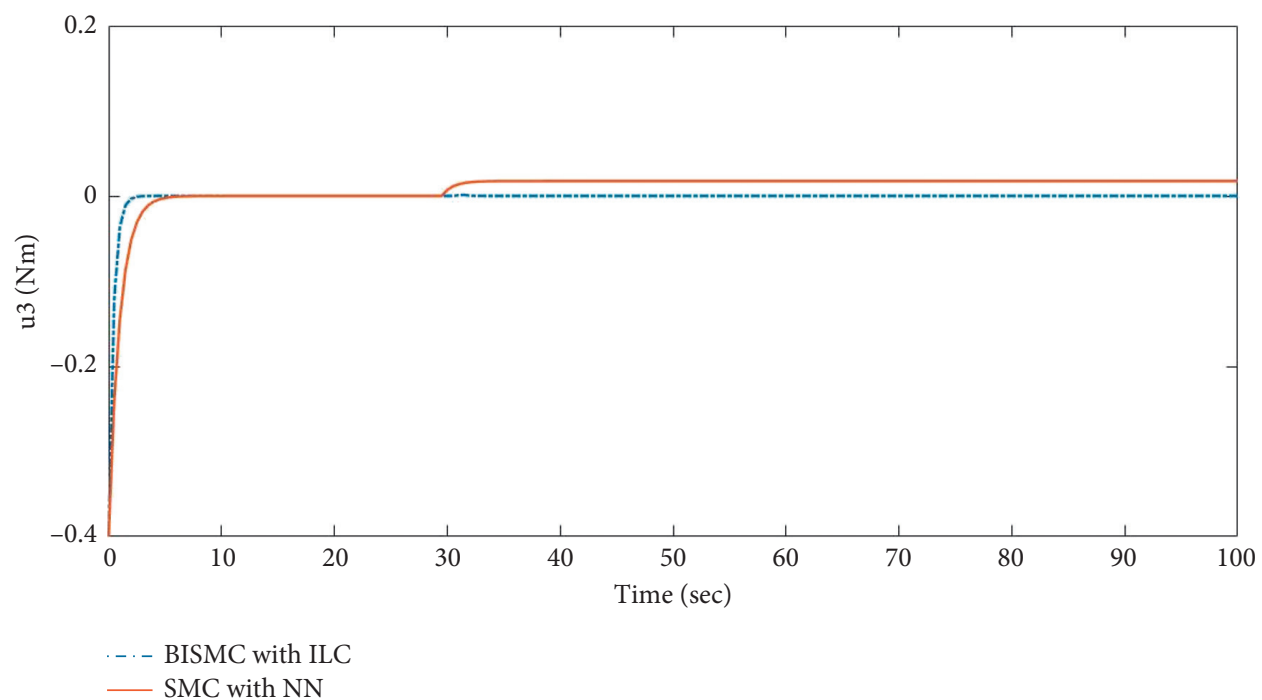

Figure 11: Control input $u_{3}(\mathrm{~nm})$. 


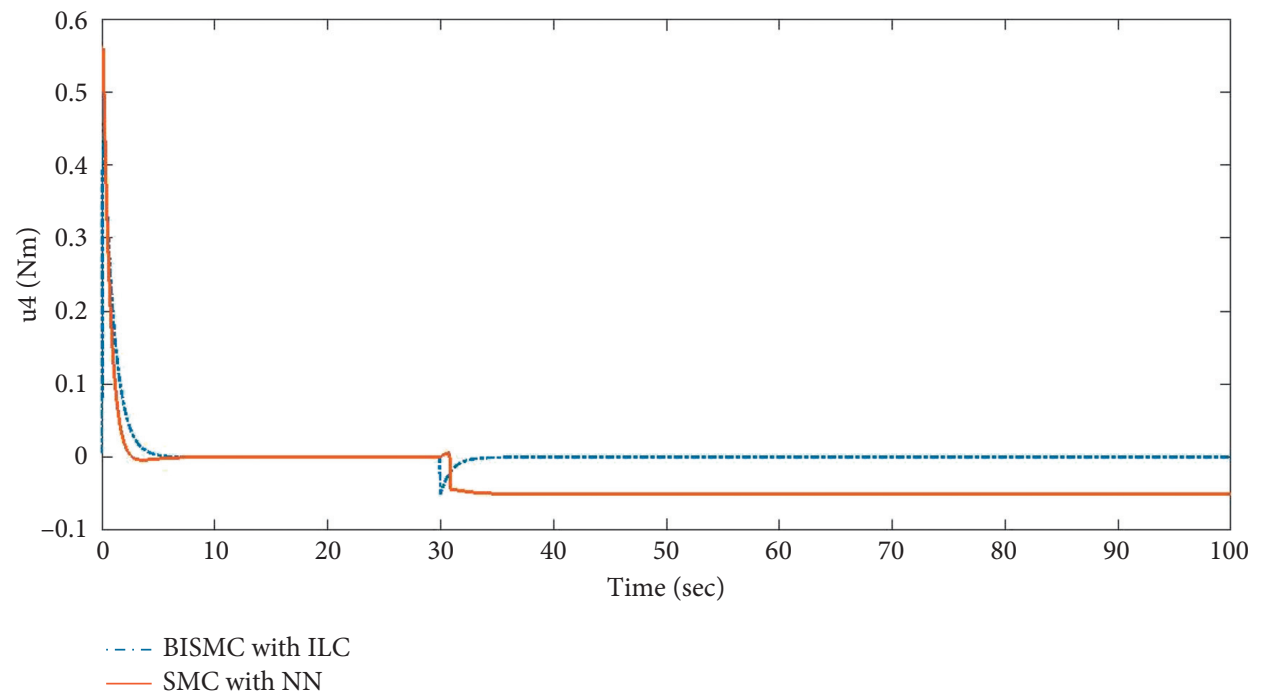

FIGURE 12: Control input $u_{4}(\mathrm{~nm})$.

TABLe 2: Numerical index.

\begin{tabular}{lcc}
\hline Name of index & BISMC with ILC & SMC with NN \\
\hline $\int x_{3}^{2}+Z_{3}$ & 1.570 & 2.107 \\
$\int x_{4}^{2}+Z_{4}$ & 1.2662 & 1.470 \\
$\int x_{5}^{2}+Z_{5}$ & 0.7384 & 1.194 \\
$\int x_{6}^{2}+Z_{6}$ & 8.4865 & 10.485 \\
\hline
\end{tabular}

$\zeta_{1}=0.6$. It is crystal clear that the controllers $u_{2}, u_{3}$, and $u_{4}$ are affected by $u_{1}$.

Figures (3)-(5) illustrate the translational states tracking such as $x$-, $y$-, and $z$-direction. From the results, it can be concluded that by using the proposed scheme based on BISMC and ILCA approach, system states have a better tracking performance compared with [10]. It is obvious that the unknown actuator loss of effectiveness fault and bias fault at $s=30$ has a more destructive effect on [10] because the neural network cannot accurately estimate the unknown rotor failure in comparison with ILCA.

Figures (6)-(8) show the roll, pitch, and yaw angle for both methods. It is crystal clear that the mentioned FTCS has better stability for the states at $s=30$ despite the stuck at $60 \%$ of its desired value of rotor 1 . The integral terms in the sliding surface and accurate estimation in terms of unknown fault input ensured that the states by considering the mentioned control strategy have good performance to get back to the reference values after the occurrence of the fault.

The total control inputs for the system under the fault actuator are shown in Figures (9)-(12). From these figures, it can be concluded that after the rotor fault, the control signals change at $=30 \mathrm{~s}$ for both control strategies but the mentioned FTCS based on BISMC and ILC has lower fluctuation in comparison with [10]. To validate the effectiveness of the proposed controller, numerical indexes are shown in Table 2 for both methods. The values of indexes in our control strategy are less than the comparative method. It means that our control strategy is faster than the another method to force the error of states toward zero.

\section{Conclusion}

In this paper, a FTCS based on BISMC was designed and analyzed for both rotational and translational subsystems of quadrotor UAVs in the presence of actuator faults and external disturbances. The loss of effectiveness fault and bias fault were considered as actuator faults in the dynamic. Next, ILCA was presented to calculate the unknown faults input. The Lyapunov theory was proved that the mentioned FTCA method can guarantee the stability of the closed-loop subsystems of quadrotor UAVs. The simulation results show that the FTCS by considering BISMC and ILCA has better performance in terms of high accuracy, better tracking, and estimation in comparison with another method [10].

\section{Appendix}

$z_{4}=x_{4}-x_{4 d}$ is defined as altitude error, and the time derivation can be written as $\dot{z}_{4}=\dot{x}_{4}-\dot{x}_{4 d}$.

By considering positive Lyapunov function and the time derivation as $v_{3}=1 / 2 z_{4}^{2}$ and $\dot{v}_{3}=z_{4} \dot{z}_{4}$, respectively, we have

$$
\dot{v}_{3}=z_{4}\left(\dot{x}_{4}-\dot{x}_{4 d}\right) \text {. }
$$

Define $z_{10}=\dot{x}_{4}-a_{2}$, where $a_{2}$ is a virtual control input. Then, $\dot{v}_{3}$ can be rewritten as follows:

$$
\dot{v}_{3}=z_{4} z_{10}+z_{4}\left(a_{2}-\dot{x}_{4 d}\right) \text {. }
$$

To make the stability of equation (A.2), $a_{2}$ is defined as follows:

$$
a_{2}=\dot{x}_{4 d}-c_{2} z_{4},
$$

where $c_{2}>0$. Next, by replacing equation (A.3) into equation (A.2), $\dot{v}_{3}$ can be computed as follows:

$$
\dot{v}_{3}=z_{4} z_{10}+z_{4}\left(\dot{x}_{4 d}-c_{2} z_{4}-\dot{x}_{4 d}\right)=-c_{2} z_{4}^{2}+z_{4} z_{10}
$$


The integral sliding mode surface for the translation subsystem is selected as follows:

$$
s_{2}=z_{10}+k_{2} \int z_{4} .
$$

By the combination of the switching law as $\dot{s}_{2}=-\varepsilon s_{2}-$ $\operatorname{sgn}\left(s_{2}\right)$ with the time derivation of equation (A.5),

$$
\dot{z}_{10}+k_{2} z_{4}=-\varepsilon s_{2}-\operatorname{sgn}\left(s_{2}\right)
$$

Replacing equation (A.4) with equation (A.6), the control torque $\delta_{2}$ is calculated as follows:

$$
\delta_{2}=\frac{I_{x}}{L b}\left[-\left(k_{2}+c_{2}\right) z_{4}-\varepsilon s_{2}-\operatorname{sgn}\left(s_{2}\right)+\dot{x}_{10 d}+\frac{d_{\varphi}}{I_{x}}-x_{11} \Omega_{r} \frac{J_{r}}{I_{x}}-x_{11} x_{12} \frac{I_{y}-I_{z}}{I_{y}}\right]-\frac{U_{2 d}}{e_{2}}-\frac{\zeta_{2}}{e_{2}} .
$$

A positive Lyapunov function is defined for stability analysis as follows:

$$
v_{4}=\frac{1}{2} v_{3}^{2}+\frac{1}{2} s_{2}^{2}
$$
follows:

The time derivation of equation (A.8) is calculated as

$$
\dot{v}_{4}=v_{3} \dot{v}_{3}+s_{2} \dot{s}_{2}
$$

and

$$
\dot{v}_{4}=v_{3} \dot{v}_{3}+s_{2}\left(x_{11} x_{12} \frac{I_{y}-I_{z}}{I_{y}}+x_{11} \Omega_{r} \frac{J_{r}}{I_{x}}-\frac{d_{\varphi}}{I_{x}}+\frac{L b}{I_{x}}\left(e_{2} \delta_{2}+\zeta_{2}+U_{2 d}\right)-\frac{d_{\varphi}}{m}\right) .
$$

By replacing equation (A.7) into equation (A.10), the necessary condition of the Lyapunov theorem is verified $\left(\dot{v}_{4}<0\right)$.

$z_{5}=x_{5}-x_{5 d}$ is defined as altitude error, and the time derivation can be written as $\dot{z}_{5}=\dot{x}_{5}-\dot{x}_{5 d}$.

By considering positive Lyapunov function and the time derivation as $v_{5}=(1 / 2) z_{5}^{2}$ and $\dot{v}_{5}=z_{5} \dot{z}_{5}$, respectively, we have

$$
\dot{v}_{5}=z_{5}+\left(\dot{x}_{5}-\dot{x}_{5 d}\right) \text {. }
$$

Define $z_{11}=\dot{x}_{5}-a_{3}$, where $a_{3}$ is a virtual control input. Then, $\dot{v}_{5}$ can be rewritten as follows:

$$
\dot{v}_{5}=z_{5} z_{11}+z_{5}\left(a_{3}-\dot{x}_{5 d}\right) \text {. }
$$

To make the stability of equation (A.12), $a_{3}$ is defined as follows:

$$
a_{3}=\dot{x}_{5 d}-c_{3} z_{5}
$$

where $c_{3}>0$. Next, by replacing equation (A.13) into equation (A.12), $\dot{v}_{5}$ can be computed as follows:

$$
\dot{v}_{5}=z_{5} z_{11}+z_{5}\left(\dot{x}_{5 d}-c_{3} z_{5}-\dot{x}_{5 d}\right)=-c_{3} z_{5}^{2}+z_{5} z_{11} .
$$

The integral sliding mode surface for the translation subsystem is selected as follows:

$$
s_{3}=z_{11}+k_{3} \int z_{5} \text {. }
$$

By the combination of the switching law as $\dot{s}_{3}=-\varepsilon s_{3}-$ $\operatorname{sgn}\left(s_{3}\right)$ with the time derivation of equation (A.15),

$$
\dot{z}_{11}+k_{3} z_{5}=-\varepsilon s_{3}-\operatorname{sgn}\left(s_{3}\right) \text {. }
$$

Replacing equation (A.14) with equation (A.16), the control torque $\delta_{3}$ is calculated as follows:

$$
\delta_{3}=\frac{I_{y}}{L b}\left[-\left(k_{3}+c_{3}\right) z_{5}-\varepsilon s_{3}-\operatorname{sgn}\left(s_{3}\right)+\dot{x}_{12 d}+\frac{d_{\theta}}{I_{y}}-x_{10} \Omega_{r} \frac{J_{r}}{I_{y}}-x_{10} x_{12} \frac{I_{z}-I_{x}}{I_{y}}\right]-\frac{U_{3 d}}{e_{3}}-\frac{\zeta_{3}}{e_{3}} .
$$

The positive Lyapunov function is defined for stability analysis as follows:

$$
v_{6}=\frac{1}{2} v_{5}^{2}+\frac{1}{2} s_{3}^{2}
$$

The time derivation of equation (A.18) is calculated as follows:

$$
\dot{v}_{6}=v_{5} \dot{v}_{5}+s_{3} \dot{s}_{3} \text {, }
$$

and 


$$
\dot{v}_{6}=v_{5} \dot{v}_{5}+s_{3}\left(-\frac{d_{\theta}}{I_{y}}+x_{10} \Omega_{r} \frac{J_{r}}{I_{y}}+x_{10} x_{12} \frac{I_{z}-I_{x}}{I_{y}}+\frac{L b}{I_{y}}\left(e_{3} \delta_{3}+\zeta_{3}+U_{3 d}\right)-\frac{d_{\theta}}{m}\right) .
$$

By replacing equation (A.17) into equation (A.20), the necessary condition of the Lyapunov theorem is verified $\left(\dot{v}_{6}<0\right)$.

$z_{6}=x_{6}-x_{6 d}$ is defined as altitude error, and the time derivation can be written as $\dot{z}_{6}=\dot{x}_{6}-\dot{x}_{6 \mathrm{~d}}$.

By considering positive Lyapunov function and the time derivation as $v_{7}=(1 / 2) z_{6}^{2}$ and $\dot{v}_{7}=z_{6} \dot{z}_{6}$, respectively, we have

$$
\dot{v}_{7}=z_{6}\left(\dot{x}_{6}-\dot{x}_{6 d}\right)
$$

Define $z_{12}=\dot{x}_{6}-a_{4}$, where $a_{4}$ is a virtual control input. Then, $\dot{v}_{7}$ can be rewritten as follows:

$$
\dot{v}_{7}=z_{6} z_{12}+z_{6}\left(a_{4}-\dot{x}_{6 d}\right) \text {. }
$$

To make the stability of equation (A.22), $a_{4}$ is defined as follows:

$$
a_{4}=\dot{x}_{6 d}-c_{4} z_{6} \text {, }
$$

$$
\delta_{4}=\frac{I_{z}}{d}\left[-\left(k_{4}+c_{4}\right) z_{6}-\varepsilon s_{4}-\operatorname{sgn}\left(s_{4}\right)+\dot{x}_{12 d}+\frac{d_{\Psi}}{I_{z}}-x_{11} x_{12} \frac{I_{x}-I_{y}}{I_{z}}\right]-\frac{U_{4 d}}{e_{4}}-\frac{\zeta_{4}}{e_{4}} .
$$

Positive Lyapunov function is defined for stability analysis as follows:

$$
v_{8}=\frac{1}{2} v_{7}^{2}+\frac{1}{2} s_{4}^{2}
$$

where $c_{4}>0$. Next, by replacing equation (A.23) into equation (A.22), $\dot{v}_{7}$ can be computed as follows:

$$
\dot{v}_{7}=z_{6} z_{12}+z_{6}\left(\dot{x}_{6 d}-c_{4} z_{6}-\dot{x}_{6 d}\right)-c_{4} z_{6}^{2}+z_{6} z_{12} .
$$

The integral sliding mode surface for the translation subsystem is selected as follows:

$$
s_{4}=z_{12}+k_{4} \int z_{6}
$$

By the combination of the switching law as $\dot{s}_{4}=-\varepsilon s_{4}-$ $\operatorname{sgn}\left(s_{4}\right)$ with the time derivation of equation (A.25),

$$
\dot{z}_{12}+k_{4} z_{6}=-\varepsilon s_{4}-\operatorname{sgn}\left(s_{4}\right) \text {. }
$$

Replacing equation (A.24) with equation (A.26), the control torque $\delta_{4}$ is calculated as follows: follows:

The time derivation of equation (A.28) is calculated as

$$
\dot{v}_{8}=v_{7} \dot{v}_{7}+s_{4} \dot{s}_{4},
$$

and

$$
\dot{v}_{8}=v_{7} \dot{v}_{7}+s_{4}\left(-\frac{d_{\Psi}}{I_{z}}+x_{11} x_{12} \frac{I_{x}-I_{y}}{I_{z}}+\frac{d}{I_{z}}\left(e_{4} \delta_{4}+\zeta_{4}+U_{4 d}\right)-\frac{d_{\Psi}}{m}\right) \text {. }
$$

By replacing equation (A.27) into equation (A.30), the necessary condition of the Lyapunov theorem is verified $\left(\dot{v}_{8}<0\right)$.

\section{Data Availability}

The source code data used to support the findings of this study are available from the corresponding author upon request.

\section{Conflicts of Interest}

The authors declare that they have no conflicts of interest.

\section{References}

[1] Z. Zhu and S. Cao, "Back-stepping sliding mode control method for quadrotor UAV with actuator failure," The Journal of Engineering, vol. 2019, no. 12, pp. 8374-8377, 2019.
[2] F. J. Niroumand and A. Fakharian, "Trajectory tracking via adaptive nonlinear control approach for a quadrotor MAV," in Proceedings of the 2015 AI \& Robotics (IRANOPEN), pp. 1-7, Qazvin, Iran, April 2015.

[3] F. Zheng, Z. Zhen, and H. Gong, "Observer-based backstepping longitudinal control for carrier-based UAV with actuator faults," Journal of Systems Engineering and Electronics, vol. 28, no. 2, pp. 322-377, 2017.

[4] S. Zeghlache, A. Djerioui, L. Benyettou, T. Benslimane, H. Mekki, and A. Bouguerra, "Fault tolerant control for modified quadrotor via adaptive type-2 fuzzy backstepping subject to actuator faults," ISA Transactions, vol. 95, pp. 330-345, 2019.

[5] F. J. Niroumand, A. Fakharian, and M. S. Seyedsajadi, "Fuzzy integral backstepping control approach in attitude stabilization of a quadrotor UAV," in Proceedings of the 2013 13th Iranian Conference on Fuzzy Systems (IFSC), pp. 1-6, Qazvin, Iran, August 2013. 
[6] Z. Yu, Y. Zhang, B. Jiang et al., "Decentralized fractionalorder backstepping fault-tolerant control of multi-UAVs against actuator faults and wind effects," Aerospace Science and Technology, vol. 104, Article ID 105939, 2020.

[7] S. Mallavalli and A. Fekih, "A fault-tolerant control design for actuator fault mitigation in quadrotor UAVs," in Proceedings of the American Control Conference (ACC), pp. 5111-5116, Philadelphia, PA, USA, July 2019.

[8] Z. Song and K. Sun, "Adaptive fault tolerant control for a small coaxial rotor unmanned aerial vehicles with partial loss of actuator effectiveness," Aerospace Science and Technology, vol. 88, pp. 362-379, 2019.

[9] Q. Shen, C. Yue, C. H. Goh, and D. Wang, "Active faulttolerant control system design for spacecraft attitude maneuvers with actuator saturation and faults," IEEE Transactions on Industrial Electronics, vol. 66, no. 55, pp. 3763-3772, 2018.

[10] X. Shi, Y. Cheng, C. Yin, H. Shi, and X. Huang, "Actuator fault-tolerant controlling using adaptive radical basis function neural network SMC for quadrotor UAV," in Proceedings of the 2019 Chinese Control and Decision Conference (CCDC), pp. 5409-5414, Nanchang, China, June 2019.

[11] H. Nemati, M. Bando, and S. Hokamoto, "Chattering attenuation sliding mode approach for nonlinear systems," Asian Journal of Control, vol. 19, no. 4, pp. 1519-1531, 2017.

[12] C. Yin, X. Huang, Y. Chen, S. Dadras, S.-M. Zhong, and Y. Cheng, "Fractional-order exponential switching technique to enhance sliding mode control," Applied Mathematical Modelling, vol. 44, pp. 705-726, 2017.

[13] Z. Zheng, Y. Q. Xia, and M. Y. Fu, “Attitude stabilization of rigid spacecraft with finite-time convergence," International Journal of Robust and Nonlinear Control, vol. 21, pp. 686-702, 2011.

[14] B. Barikbin and A. Fakharian, "Trajectory tracking for quadrotor UAV transporting cable-suspended payload in wind presence," Transactions of the Institute of Measurement and Control, vol. 41, no. 5, pp. 1243-1255, 2019.

[15] S. Ullah, K. Iqbal, and M. F. Malik, "Fault-tolerant sliding mode control of a quadrotor UAV with delayed feedback," International Journal of Mechanical Engineering and Robotics Research, vol. 9, no. 1, 2020.

[16] Z. Hou, P. Lu, and Z. Tu, "Nonsingular terminal sliding mode control for a quadrotor UAV with a total rotor failure," Aerospace Science and Technology, vol. 98, Article ID 105716, 2020.

[17] N. P. Nguyen and S. K. Hong, "Fault-tolerant control of quadcopter UAVs using robust adaptive sliding mode approach," Energies, vol. 12, no. 6, p. 1139, 2019.

[18] B. Wang, X. Yu, L. Mu, and Y. Zhang, "A dual adaptive faulttolerant control for a quadrotor helicopter against actuator faults and model uncertainties without overestimation," Aerospace Science and Technology, vol. 99, Article ID 105744, 2020.

[19] W. Hao and B. Xian, "Nonlinear fault-tolerant control for a tri-rotor UAV against rear servo's stuck fault," in Proceedings of the 36th Chinese Control Conference (CCC), pp. 7109-7114, Dalian, China, July 2017.

[20] S. Mallavalli and A. Fekih, "Sliding model-based fault-tolerant control designs for quadrotor UAVs-A comparative study," in Proceedings of the 13th IEEE International Conference on Control \& Automation (ICCA), pp. 154-159, Ohrid, Macedonia, July 2017.

[21] S. Mallavalli and A. Fekih, "Dynamic inversion and integral terminal sliding mode-based control design for a quadrotor
UAV," in Proceedings of the 14th International Conference on Control and Automation (ICCA), pp. 300-305, Anchorage, AK, USA, June 2018.

[22] Z. Yu, Z. Liu, Y. Zhang, Y. Qu, and C. Y. Su, "Distributed finite-time fault-tolerant containment control for multiple unmanned aerial vehicles," IEEE Transactions on Neural Networks and Learning Systems, vol. 31, no. 6, pp. 2077-2091, 2019.

[23] R. Tayari, A. B. Brahim, and F. B. Hmida, "Actuator fault tolerant control design for linear parameter varying systems," in Proceedings of the International Conference on Advanced Systems and Emergent Technologies (IC_ASET), pp. 17-21, Hammamet, Tunisia, March 2019.

[24] W. Gong, J. Zhang, B. Li, and Y. Yang, "Integral-type sliding mode based fault-tolerant attitude stabilization of a quadrotor UAV," in Proceedings of the International Symposium in Sensing and Instrumentation in IoT Era (ISSI), pp. 1-6, Shanghai, China, September 2018.

[25] Z. Yu, Y. Zhang, Z. Liu, Y. Qu, and C. Y. Su, "Distributed adaptive fractional-order fault-tolerant cooperative control of networked unmanned aerial vehicles via fuzzy neural networks," IET Control Theory \& Applications, vol. 13, no. 17, pp. 2917-2929, 2019.

[26] P. Cheng, Z. Gao, M. Qian, and J. Lin, “Active fault-tolerant control design for UAV using nonsingular fast terminal sliding mode approach," in Proceedings of the Chinese Control and Decision Conference (CCDC), pp. 292-297, Shenyang, China, June 2018.

[27] J. Wang, C. Yang, J. Xia, Z.-G. Wu, and H. Shen, "Observerbased sliding mode control for networked fuzzy singularly perturbed systems under weighted try-once-discard protocol," IEEE Transactions on Fuzzy Systems, 2021.

[28] J. Wang, C. Yang, H. Shen, J. Cao, and L. Rutkowski, "Slidingmode control for slow-sampling singularly perturbed systems subject to markov jump parameters," IEEE Transactions on Systems, Man, and Cybernetics: Systems, 2020.

[29] J. Dong and B. He, "Novel fuzzy PID-type iterative learning control for quadrotor UAV," Sensors, vol. 19, no. 1, p. 24, 2019.

[30] Y. Wang, J. Shi, D. Zhou, and F. Gao, "Iterative learning faulttolerant control for batch processes," Industrial \& Engineering Chemistry Research, vol. 45, no. 26, pp. 9050-9060, 2006.

[31] G. Liu and Z. Hou, "Cooperative adaptive iterative learning fault-tolerant control scheme for multiple subway trains," IEEE Transactions on Cybernetics, 2020.

[32] N. Raj, A. Simha, M. Kothari, and R. N. Banavar, "Iterative learning based feedforward control for transition of a biplanequadrotor tailsitter UAS," in Proceedings of the 2020 IEEE International Conference on Robotics and Automation (ICRA), pp. 321-327, Paris, France, August 2020.

[33] A. Hock and A. P. Schoellig, "Distributed iterative learning control for multi-agent systems," Autonomous Robots, vol. 43, no. 8, pp. 1989-2010, 2019.

[34] A. Schöllig and R. D’Andrea, "Optimization-based Iterative learning control for trajectory tracking," in Proceedings of the European Control Conference (ECC), pp. 1505-1510, Budapest, Hungary, August 2009.

[35] D. Allahverdy, A. Fakharian, and M. B. Menhaj, "Backstepping integral sliding mode control with iterative learning control algorithm for quadrotor UAVs," Journal of Electrical Engineering \& Technology, vol. 14, no. 6, pp. 2539-2547, 2019.

[36] H. A. Foudeh, P. Luk, and J. Whidborne, "Application of norm optimal iterative learning control to quadrotor unmanned aerial vehicle for monitoring overhead power system," Energies, vol. 13, no. 12, p. 3223, 2020. 
[37] W. Giernacki, "Iterative learning method for in-flight autotuning of UAV controllers based on basic sensory information," Applied Sciences, vol. 9, no. 4, p. 648, 2019.

[38] Z. Zhao, J. Wang, Y. Chen, and S. Ju, "Iterative learning-based formation control for multiple quadrotor unmanned aerial vehicles," International Journal of Advanced Robotic Systems, vol. 17, no. 2, Article ID 1729881420911520, 2020.

[39] D. A. Bristow, M. Tharayil, and A. G. Alleyne, "A survey of iterative learning control," IEEE Control Systems, vol. 26, no. 3, pp. 96-114, 2006.

[40] D. Allahverdy and A. Fakharian, "Active fault-tolerant control system for a swash mass helicopter using back-stepping approach," in Proceedings of the Institution of Mechanical Engineers, Part I: Journal of Systems and Control Engineering, Article ID 09596518211022903, 2021. 